\title{
S-06: Effect of Increased Cadence on Neuromuscular Response and Lower Limb Kinematics in Middle Distance Runners
}

\author{
Amrinder Singh, Nupur Hisaria, Shweta Shenoy, Jasmine Chawla, Jaspal Sandhu
}

\begin{abstract}
AIM: To examine the differences in the neuromuscular and kinematic response in middle distance runners when the step rate was increased to $5 \%$ and $10 \%$ respectively of the preferred step rate.

MATERIALS AND METHODOLOGY: 80 middle distance runners (40 males and 40 females) participated in the study. EMG of 8 muscles (rectus femoris, medial and lateral hamstrings, medial gastrocnemius, tibialis anterior, gluteus medius and maximus), kinematic changes at the lower limb joints during stance and swing phase of running, foot pressure readings and spatiotemporal gait pa-rameters were recorded as each participant ran at their preferred speed, $5 \%$ increase in preferred speed and $10 \%$ increase in their preferred speed while keeping the treadmill speed constant.

RESULTS: Kinematic analysis showed an increased knee flexion angle at initial contact $(p<$ $0.001)$ and decreased knee flexion angle during swing phase $(p<0.001)$, Ankle showed reduced dorsiflex-ion angle at initial contact $(p<0.001)$ and the hip showed less peak flexion $(p<0.001)$ across step rate conditions.

With an increase in step rate, a fore foot strike running pattern was seen. Muscle activity was signif-icantly increased during the late swing and pre-swing at $10 \%$ of the preferred speed $(p<0.001)$, with less significant changes during $5 \%$ of the preferred speed. During loading response, significant de-crease in muscle activity $(p<0.05)$ was found between $5 \%$ and $10 \%$ increase of preferred speed.

CONCLUSION: Increased cadence showed increased muscle activation and a forefoot strike running pattern. The kinematic changes documented along with the neuromuscular response across the step rate conditions could be beneficial to runners for rehabilitation and injury prevention.
\end{abstract}

Available at: http://journalofsportsmedicine.org and http://dx.doi.org/10.5152/tjsm.2017.010

(C2017 Türkiye Spor Hekimleri Derneği. Tüm hakları saklıdır. 